\title{
CALET measurements with cosmic nuclei: expected performances of tracking and charge identification
}

\author{
Paolo Brogi* \\ University of Siena and INFN Pisa \\ E-mail: p.brogi@cern.ch \\ Pier Simone Marrocchesi \\ University of Siena and INFN Pisa \\ E-mail: marrocchesi@pi.infn.it

\section{Paolo Maestro} \\ University of Siena and INFN Pisa \\ E-mail: paolo.maestro@pi.infn.it

\section{Nicola Mori} \\ University of Florence and INFN Florence \\ E-mail: nicola.moriefi.infn.it
}

CALET is a space mission currently in the final phase of preparation for a launch to the International Space Station (ISS), where it will be installed on the Exposed Facility of the Japanese Experiment Module (JEM-EF). In addition to high precision measurements of the electron spectrum, CALET will also perform long exposure observations of cosmic nuclei from proton to iron and will detect trans-iron elements with a dynamic range up to $Z=40$. The energy measurement relies on two calorimeter systems: a fine grained imaging calorimeter (IMC) followed by a total absorption calorimeter (TASC) for a total thickness of $30 X_{0}$ and 1.3 proton interaction length. A dedicated module (a charge detector, $\mathrm{CHD}$ ), placed at the top of the apparatus, identifies the atomic number $\mathrm{Z}$ of the incoming cosmic ray. In this paper, the IMC performances in providing tracking capabilities and a redundant charge measurement by multiple $\frac{d E}{d x}$ samples are studied for the case of proton and He identification with a preliminary version of the analysis. The CALET mission is funded by the Japanese Space Agency (JAXA), the Italian Space Agency (ASI), and NASA.

The 34th International Cosmic Ray Conference,

30 July- 6 August, 2015

The Hague, The Netherlands

\footnotetext{
${ }^{*}$ On behalf of the CALET collaboration.
} 


\section{The CALET experiment}

CALET is an electron telescope in preparation for a launch to the International Space Station (ISS), where it will be installed on the Exposure Facility of the Japanese Experiment Module (JEM-EF). With observations over a period of five years, it will be able to detect the presence of possible nearby sources of high energy electrons and search for signatures of dark matter. It will also monitor gamma ray transients with a dedicated Gamma-ray Burst Monitor (CGBM) and study solar modulation. CALET will perform long exposure observations of cosmic nuclei from proton to iron and will detect trans-iron elements with a dynamic range up to $\mathrm{Z}=40$. It will extend, by one order of magnitude in energy, the present measurements of the ratio of secondary to primary elements (e.g.: boron/carbon and sub-iron/iron), providing information about the energy dependence of cosmic-ray propagation in the galaxy. CALET is an all-calorimetric instrument, with a total thickness of 30 radiation length $\left(\mathrm{X}_{0}\right)$ and 1.3 proton interaction length $\left(\lambda_{I}\right)$, preceded by a particle identification system. The energy measurement relies on two kinds of calorimeters: a fine grained pre-shower, known as imaging calorimeter (IMC), followed by a total absorption calorimeter (TASC). The TASC measures the total energy of the incident particle and discriminates electrons and gamma-rays from hadrons. It is composed of 12 layers of Lead Tungstate (PWO) "logs", each with dimensions $20 \mathrm{~mm}(\mathrm{H}) \times 19 \mathrm{~mm}(\mathrm{~W}) \times 326 \mathrm{~mm}(\mathrm{~L})$. Alternate layers are oriented perpendicular to each other to provide $\mathrm{x}, \mathrm{y}$ coordinates of the shower core. The total thickness of the TASC is about $27 \mathrm{X}_{0}$ at normal incidence. In order to identify individual chemical elements in the cosmic-ray flux, a Charge Detector (CHD) is positioned at the top of the CALET instrument to provide a measurement of the electric charge $[1,2,3]$. Calibration and test of the instrument took place at CERN SPS during five campaigns in 2010, 2011, 2012, 2013 and 2015 with beams of electrons, protons and relativistic ions.

\section{Tracking with the IMC detector}

The IMC images the early shower profile with a fine granularity by using $1 \mathrm{~mm}^{2}$ scintillating fibers individually readout by multi-anode photomultipliers (MAPMT). It consists of 7 layers of tungsten plates, each separated by 2 layers of scintillating fibers ( $\mathrm{SciFi}$ ) with square cross section, arranged in belts along the $\mathrm{x}$ and $\mathrm{y}$ direction and is capped by an additional x,y SciFi layer pair. The transverse dimensions of the IMC are approximately $45 \mathrm{~cm}$ by $45 \mathrm{~cm}$. The total thickness of the IMC is equivalent to $3 \mathrm{X}_{0}$. The first 5 tungsten-SciFi layers sample the shower every 0.2 $\mathrm{X}_{0}$ while the following 2 layers provide $1.0 \mathrm{X}_{0}$ sampling. The IMC is designed to: (i) separate the incident particles; (ii) determine the starting point of the shower; (iii) reconstruct the incident particle trajectory, and (iv) provide a redundant measurement of the particle's charge.

Track identification and reconstruction is of utmost importance for CALET, not only to detect the direction of the incident particle, but to improve the data analysis in a number of cases. As an example, the standard proton rejection algorithm relies on topological calorimetric variables as, for instance, the width of the shower with respect to the particle trajectory. Moreover, the use of multiple $\frac{d E}{d x}$ sample in the IMC fibres to improve the overall charge reconstruction relies on an adequate primary track identification. For this purpose, a Kalman filter [4] algorithm is implemented. The linear Kalman filter is a hybrid technique for simultaneous track finding and fitting. In its combinatorial formulation [5] it provides robust track identification which can deal with spurious hits and "holes" along the track due to inefficiencies. 


\subsection{Kalman filter Implementation}

The track finding problem in CALET can be decomposed into two independent 2D problems in the $\mathrm{XZ}$ and $\mathrm{YZ}$ views, respectively. The local hit point information is $2 \mathrm{D}$ since each single detector layer is segmented in either $\mathrm{X}$ or $\mathrm{Y}$ direction, and placed at a different $\mathrm{Z}$ coordinate. The developed algorithm is thus a generic $2 \mathrm{D}$ Kalman filter for planar 1D detectors arranged along the longitudinal $\mathrm{Z}$ axis. The algorithm is fed with the $\mathrm{Z}$ positions of sensors, the $\mathrm{X}_{i}$ (or $\mathrm{Y}_{i}$ ) positions and $\sigma_{i}$ of all hits on each sensor. The algorithm then runs separately for the $\mathrm{X}$ and $\mathrm{Y}$ views to find the projections of the full 3D track, which is then reconstructed in a subsequent step from the respective projections. The two-dimensional formulation has the advantage to simplify the handling of the matrix equations and the computation of inverse matrices. Once all the possible physical tracks are found by the algorithm, a candidate primary track is identified using a userdefined criterion. This criterion is based on the energy deposit associated to the track in the TASC: the IMC reconstructed track that has the largest energy deposit in TASC is chosen as the primary candidate. For the beam test data collected in 2012, due to the limited number (3) of instrumented PWO crystals per layer this criterion is modified and a simpler one is used: the primary track is identified as the one with the largest energy deposit in the IMC.

\subsection{Tracking performance}

The IMC tracking performance depends on the incident particle species and energy, for this reason the implemented algorithm was tested on different Monte Carlo and beam test data samples, including: FLUKA simulated events of electrons, protons and ions (carbon nuclei); beam test events (TB2012) of protons, electrons and muon recorded in 2012 at CERN-SPS in the North Area. As per the simulated events, they were generated isotropically in direction and according to a spectrum covering energies from $10 \mathrm{GeV}$ to $100 \mathrm{TeV}$, following a power law with spectral index -1. For beam test data instead, the analysed samples were taken with a collimated beam of $150 \mathrm{GeV}$ muons, $250 \mathrm{GeV}$ electrons, 30,100 and $400 \mathrm{GeV}$ protons, all at normal incidence with respect to the detector planes, with the exception of the $400 \mathrm{GeV}$ protons that have an incidence angle of about $20^{\circ}$.

\subsubsection{Performance with MC simulations}

Analysis of MC data samples allow to estimate the tracking efficiency and the spatial and angular resolutions. The efficiency is calculated taking into account the whole acceptance of the CALET telescope, resulting into an estimated efficiency of about $95 \%$ for simulated protons and electrons and $99 \%$ for carbon ions. To estimate the angular resolution in both $\mathrm{XZ}$ and $\mathrm{YZ}$ views ( $\theta_{x}$ and $\theta_{y}$ respectively), residuals between MC truth and reconstructed information are computed (i.e. $\Delta \theta_{x}=\theta_{x}^{M C}-\theta_{x}^{r e c o}$ and $\Delta \theta_{y}=\theta_{y}^{M C}-\theta_{y}^{r e c o}$ ). A Point Spread Function is then calculated as the half-width of the interval centred on the mean value and containing $68.3 \%$ of the events. In a similar way, an estimate of the spatial resolution at the particle incidence point (top of the CHD) is performed. The residuals for the $x$ and $y$ coordinates are calculated as the distance between the true position $\left(x_{M C}, y_{M C}\right)$ of the incident particle at $z=0$ and the one extrapolated from the reconstructed track $\left(x_{\text {reco }}, y_{\text {reco }}\right)$. The residuals are therefore $\Delta x=x_{M C}-x_{\text {reco }}$ and $\Delta y=y_{M C}-y_{\text {reco }}$ for the $x$ and $y$ view, respectively. In order to quote a $\sigma$ value for the resolution, a $68.3 \%$ interval around the mean is defined, in analogy with the PSF. This " $z=0$ " point is chosen due to its important role to identify the CHD paddle that has been hit and which is used for charge reconstruction. An example of $\theta_{x}$ 

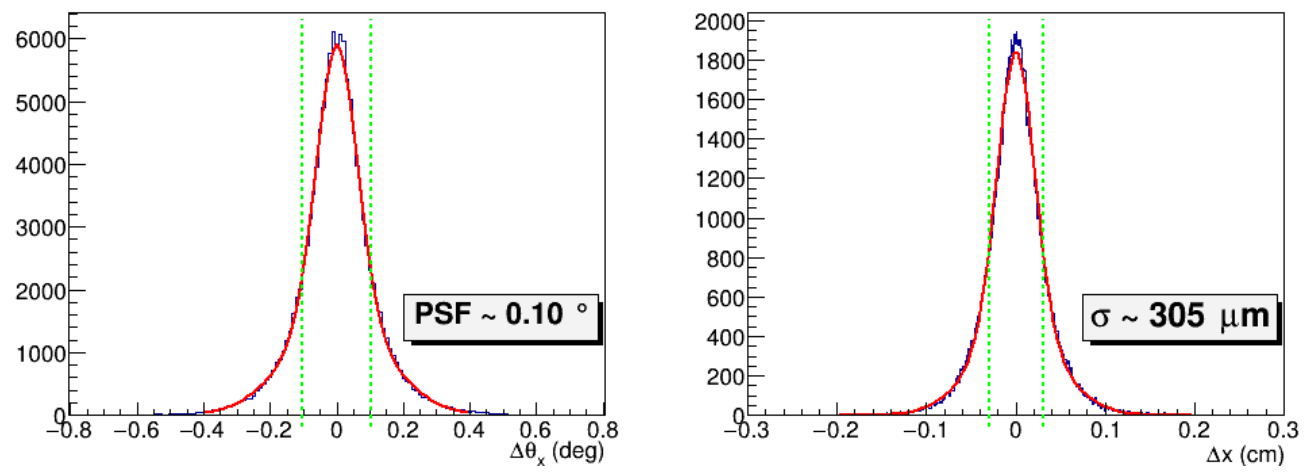

Figure 1: Left: residuals $\Delta \theta_{x}=\theta_{x}^{M C}-\theta_{x}^{\text {reco }}$ distribution. Right: $x$ coordinate residuals $\Delta x=x_{M C}-x_{\text {reco }}$ measured on the CHD surface.
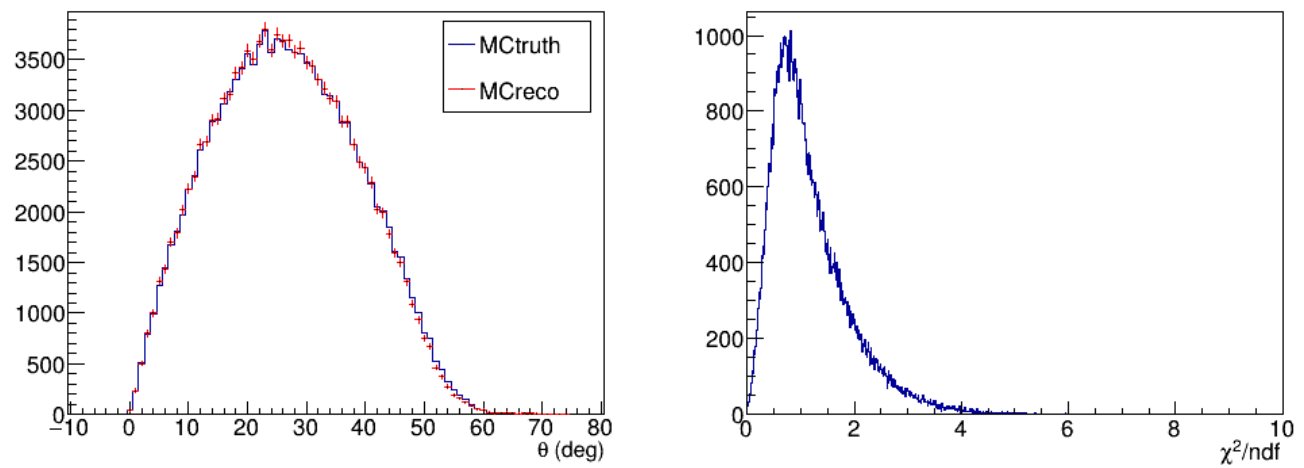

Figure 2: Left: Zenith angle of incident particle from MC (blue line) compared with reconstructed one (red marker). Right: $\chi^{2}$ distribution for reconstructed track.

and $x$ residuals for the electrons sample can be found in figure $1\left(\theta_{y}\right.$ and $y$ residuals have the same behaviour), while figure 2 shows the histogram of the reconstructed zenith angle $\theta$ superimposed to the corresponding true MC parameter. The $\chi^{2}$ distribution is shown as well. For electrons, the $\theta$ distribution is well reproduced, the $\chi^{2}$ distribution is well-behaved, and the residuals distributions are considerably narrow. Since the distributions of residuals can be approximated as a sum of two Gaussians with the same mean and different $\sigma$, a fit to this function is performed (red line in figure). For simulated protons and carbon ions events, the plots of these quantities are not shown for brevity, but have a similar behaviour, albeit the performances are different. In particular for proton and carbon, due to their hadronic nature, a distinction is made between non interacting particles (i.e. MIPs events) and interacting ones which generated a High Energy Trigger (HET) in CALET. This is the main trigger mode for CALET: it requires a large energy deposit in the middle of the detector, and it targets protons and nuclei of a few $10 \mathrm{GeV}$ to $1000 \mathrm{TeV}$. Interacting particles, not generating an HET event, are not analysed. The tracking algorithm performance for all the simulated data is summarized in the following table:

In conclusion, for MC simulated data a high tracking efficiency is achieved together with a good resolution, both in spatial and angular coordinates. As expected, the performances are worse for HET events, because track finding and also primary track identification are complicated by the much higher track and cluster multiplicity in the detector. This problem affects more proton than 


\begin{tabular}{lcccc}
\hline particle type & energy range $(\mathrm{TeV} / \mathrm{n})$ & efficiency & $P S F_{\theta_{x}-\theta_{y}}$ & $\sigma_{x-y}(\mu \mathrm{m})$ \\
\hline \hline electrons & {$[0.02,2]$} & 0.95 & $0.10^{\circ}$ & 300 \\
protons (MIP) & {$[0.01,1]$} & 0.95 & $0.10^{\circ}$ & 300 \\
protons (HET) & {$[0.01,1]$} & 0.91 & $0.42^{\circ}$ & 800 \\
protons (MIP) & {$[1,100]$} & 0.94 & $0.10^{\circ}$ & 300 \\
protons (HET) & {$[1,100]$} & 0.99 & $0.25^{\circ}$ & 700 \\
carbon nuclei (MIP) & {$[0.03,3]$} & 0.97 & $0.09^{\circ}$ & 200 \\
carbon nuclei (HET) & {$[0.03,3]$} & 0.99 & $0.14^{\circ}$ & 400 \\
\hline
\end{tabular}

Table 1: Performance of tracking algorithm tested on different MC data samples.

carbon because the track identification, via the TASC energy deposit, is found to work better for ions than for protons. Although for HET events the performance of the tracking algorithm are worse than in the case of MIPs or electrons, they turn out to be adequate for the requirements of the detector.

\subsubsection{Performance with beam test data}

After analysing the results of the tracking algorithm with MC data, a test with real data is of particular interest to validate the performance of the tracking system in a realistic situation. As mentioned in section 2.1, the primary track selection criterion is modified for the data analysis with respect to MC. For this reason, the data results are not directly comparable with the MC ones. For beam test data, detector calibration and fibres alignment are performed by using muon data samples prior to track reconstruction. Moreover an event pre-selection is needed in order to reduce analysis biases, like the ones that could occur, for instance, for events outside the detector acceptance. For this purpose, the information from another detector, positioned along the beam-line upstream of the CALET instrument, and named Beam Tracker (BT), is used. The BT detector (comprising silicon strip and silicon pixel detectors) is used to select a sample of events with a single track pointing to the IMC detector. This external tagging of events allows to estimate a real tracking efficiency, as the fraction of events well reconstructed by IMC with respect to those selected by the BT. In particular, it is found that for $250 \mathrm{GeV}$ electrons the algorithm is fully efficient, while for protons of 30, 100 and $400 \mathrm{GeV}$ the efficiency is about $96 \%$. To get an idea on how the algorithm behaves in the case of beam test data, the reconstructed $\theta_{y}$ angle for $400 \mathrm{GeV}$ protons is shown in figure 3 (left), together with the $\chi^{2}$ distribution (right). As in the case of MC data analysis, an estimate of the distribution variance is made calculating (as already defined) the PSF which is $\sim 0.26^{\circ}$, originating both from the tracking resolution and the beam intrinsic divergence. Noticeably, the $\theta_{y}$ distribution in figure 3 has a mean value of about $-20^{\circ}$. This reproduces the result of a geometrical survey of the beam test layout where the detector was rotated by a similar amount with respect to the beam line.

\section{Charge measurement with the IMC}

Using the dedicated CHarge Detector (CHD) on top of the instrument, a charge resolution of 0.15 - 0.3 charge units has been demonstrated [2,3]. Nevertheless, a redundant measurement of the charge of the incident particle is of great importance. In fact, charge tagging becomes challenging at high energies due to the albedo of backscattered particles emerging as secondaries from the 

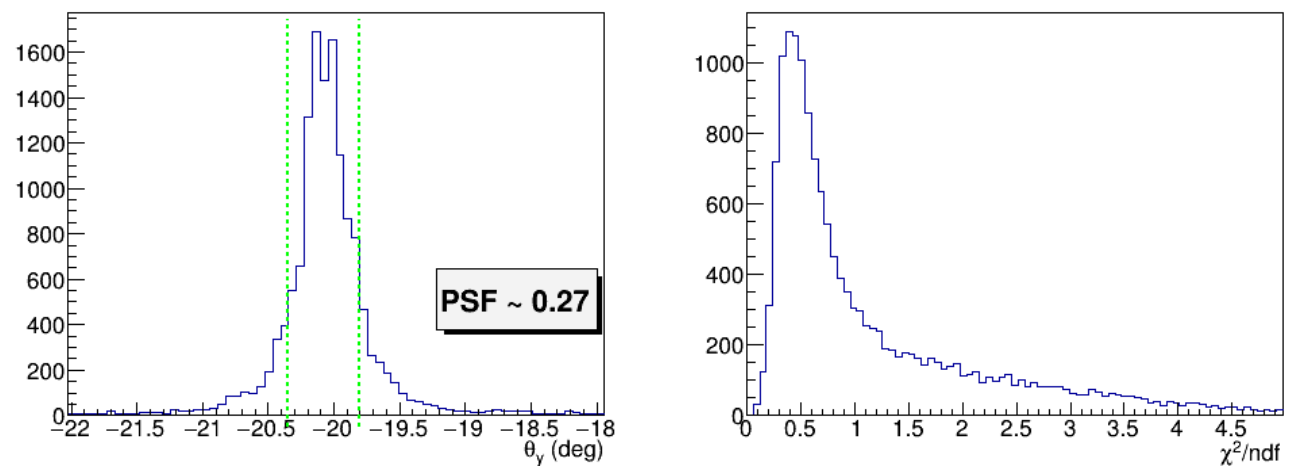

Figure 3: Distributions of reconstructed angle $\theta_{y}$ (left), $\chi^{2}$ (right) for a SPS $400 \mathrm{GeV}$ proton beam tilted by $20^{\circ}$ with respect to the detector $\mathrm{z}$ axis.

interactions in the calorimeter. A preliminary study with the IMC detector is presented in the following. As the incoming triggered particles behave as MIPs before they interact, the idea is to take advantage of the tracking information to identify the IMC fibers crossed by an incident particle of charge $\mathrm{Z}$ and to use their individual energy deposits to reconstruct the primary charge, using the proportionality of the energy loss by ionization with $Z^{2}$. The reconstruction of the charge can be divided in four consecutive steps: primary particle tracking, identification of the interaction point, $\frac{d E}{d x}$ estimate and final assessment of the charge. In the first step, extensively discussed above, it is necessary to identify the primary particle and the associated energy deposits in the IMC. The second step is mandatory because, after the interaction, the particle can no longer be considered as a MIP, therefore only the energy deposits in fibers ahead of the interaction point can be used to get a measurement of the energy loss by ionization. The idea behind the present algorithm to reconstruct the interaction point is that - after an interaction - the energy deposits along the primary track will increase considerably, therefore the goal is to identify a "discontinuity" in the energy deposit values. To do this, the algorithm proceeds in three steps. During the first step, all IMC planes are considered, starting from the top of the detector and moving to the bottom, and for each plane the average $\frac{d E}{d x}$ is calculated using all the available samples, from the top of the IMC to the layer under study. The variance is computed as well. Then, a test is performed layer by layer, checking whether the difference between the mean $\frac{d E}{d x}$ between the previous plane and at the actual one is larger than 2 times the variance (computed from the previous plane). In this case, the layer is "tagged" as a possible candidate plane in which the interaction occurred. Finally, in order to avoid that a simple fluctuation in the energy loss could lead to a misidentification of interaction point, another scan on all the layers is made to verify the existence of a point starting from which all the following planes are "tagged". In this case, it is identified as the interaction point. Once the $\frac{d E}{d x}$ sample is obtained, taking into account all the deposits ahead of the interaction point, the average energy loss is estimated requiring a minimum of 4 points in the sample and computing a truncated mean of the recorded energy deposits, with a truncation level rejecting the highest $30 \%$ deposits. After a dedicated study, this was found to be optimal for the IMC detector, because it allows to reduce the Landau tail of the $\frac{d E}{d x}$ distribution, without decreasing too much the efficiency for particle identification. Finally, the charge value is determined by cutting on the $\frac{d E}{d x}$ distribution, as described in the following. 

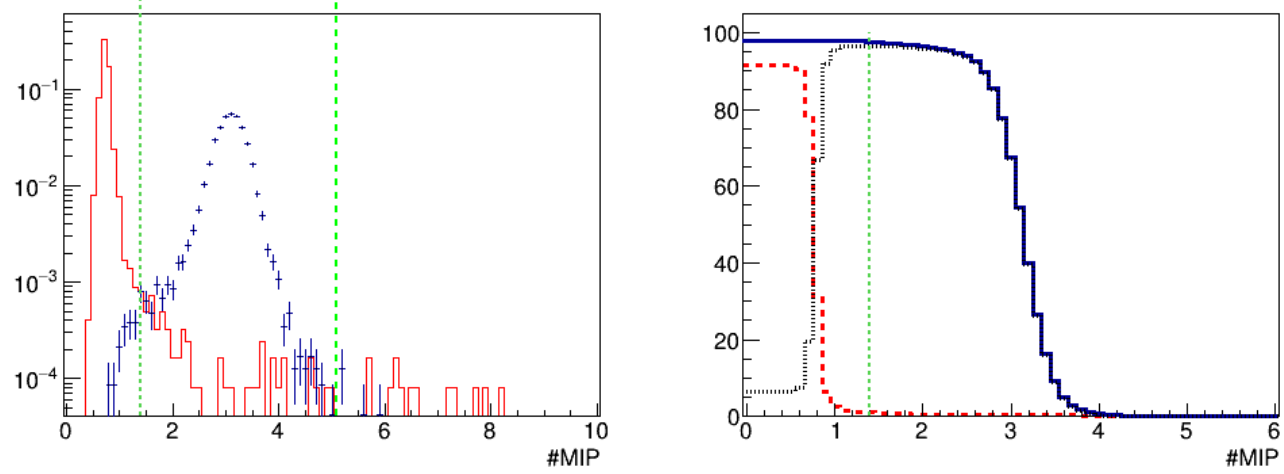

Figure 4: Left: Average $\frac{d E}{d x}$ distributions for protons (red line) and helium nuclei (blue marker), normalized with respect to their measured relative CR abundances, for incident particle energies in the range 100-180 GeV. Right: Efficiency (blue continuous line), proton contamination (red dashed line) and their difference (black dotted line) for the He selected sample depending on selection cut value, for an incident particle energy in the range $100-180 \mathrm{GeV}$.

\section{Helium and proton identification}

In view of a measurement of cosmic-ray proton and helium spectra, the charge identification procedure described in the following will focus only on these two species. For this reason the algorithm previously described is tested on two MC data samples, consisting of protons and helium nuclei, simulated with FLUKA, with energy spectra following a power law with spectral index -1 and limited to the interval from $100 \mathrm{GeV}$ to $100 \mathrm{TeV}$. To have a better characterization of the detector performance for this measurement, the data samples are divided into 12 (approximately) equally populated logarithmic bins in energy and the analysis is carried out bin per bin, separately. At this preliminary stage we decided to exclude, from the analysis reported below, those events that interact upstream of the IMC detector (e.g. in the CHD) as they need a special treatment to extract the charge information (with the combined use of the CHD detector and the IMC itself). The average $\frac{d E}{d x}$ (with a $30 \%$ truncation) is computed, as explained in the previous section, both for proton and helium. An example (relative to the first energy bin from $100 \mathrm{GeV}$ to $\sim 180 \mathrm{GeV}$ ) is shown in figure 4 (left), where the two distributions (red line for protons and blue marker for helium nuclei) are superimposed and properly normalized with respect to the expected relative CR abundances [6]. A suitable cut is then chosen to select helium nuclei from protons. The goal of the analysis is to minimize systematic errors on helium and protons selections. Because the relative abundances of helium and protons are quite similar in total energy bins, the selection cuts are optimized to maximize the difference between the helium signal and the proton contamination. In Figure 4 (right) the helium efficiency is shown (blue continuous line) as a function of the cut value, together with the proton contamination (red dashed line) and the difference between signal and contamination (black dotted line). The blue line is the percentage of reconstructed He events surviving the cut, calculated over the total number of triggers and tracked by the detector. Inefficiencies arise both from the cut and the charge reconstruction procedure (e.g. due to early interacting events). The red line is the percentage of protons that survive the cuts, contaminating the helium signal. An optimal separation cut between proton and helium distributions is then chosen as the value that maximizes the difference between helium signal and proton contamination. The value of the cut corresponds to the maximum of the black line in figure 4 (right). Moreover a signal purity is defined as the 

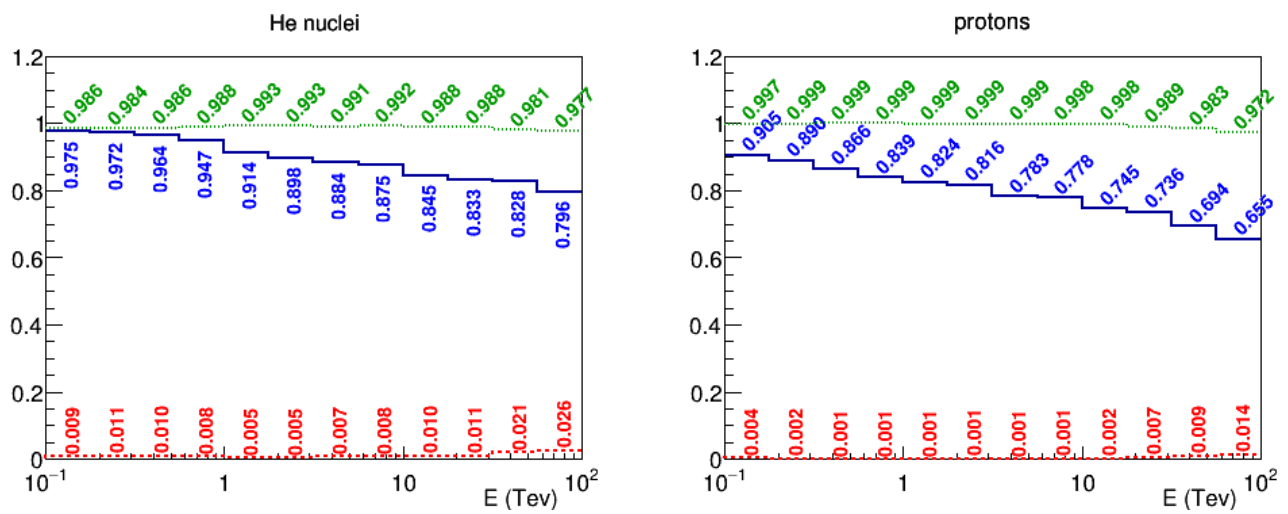

Figure 5: Efficiency (blue continuous line), contamination (red dashed line) and purity (green dotted line) of helium signal (left) or protons signal (right) respect to bin energy.

number of helium nuclei correctly identified with respect to the sum of He nuclei plus proton contamination (computed taking into account their relative abundances). This definition of the purity of helium signal includes, at present, only the contribution of protons as main contaminants. The end point of the helium distribution is estimated by fitting with a convolution function of a Landau and a Gaussian, and taking the value for which the integral from 0 to the end point is $99 \%$ of the total. The final results of the analysis are shown for each energy bin, both for helium (left) that for protons (right), in the figure 5. The quoted values represent the efficiencies (blue continuous line), purities (green dotted line) and contaminations (red dashed line) obtained with the optimal cuts from the average $\frac{d E}{d x}$ distributions calculated for each bin. It is of particular interest to notice that, for all the energy bins, a purity better than $97-98 \%$ is obtained, preserving an efficiency better then $65 \%$. This is a promising result in the direction to use the IMC detector to provide a redundant charge measurement in addition to its tracking and calorimetric functionalities.

\section{Summary}

A preliminary method of analysis has been developed to reconstruct tracks in the IMC and to provide a charge measurement of the incident particle taking advantage of multiple $\frac{d E}{d x}$ samples from the IMC layers. This would provide an independent and redundant method of particle identification to be combined with the dedicated charge measurement performed by the CHD. The IMC performances are studied in the case of the two lightest nuclei and preliminary results suggest the possibility to reach and adequate level of purity vs efficiency in the identification of $\mathrm{p}$ and $\mathrm{He}$.

\section{References}

[1] Y. Shimizu et al., Proc. of the 32nd Int.Cosmic Ray Conference (2011)

[2] P. S. Marrocchesi et al., Nucl. Instr. Meth. A 659477 (2011)

[3] S. Torii et al., "Calorimetric Electron Telescope (CALET) for the ISS", in these proceedings

[4] R. Frühwirth, Nucl. Instr. Meth. A, 262 (1987), 444-450

[5] A. Strandlie and R. Frühwirth, Rev. Mod. Phys., 82 (2010), 1419-1458

[6] D. Maurin, F. Melot and R. Taillet, A\&A 569, A32 (2014) 